\title{
Théologiques
}

\section{La traduction des textes sacrés}

\section{Alexis Nouss}

Volume 15, numéro 2, 2007

La traduction des textes sacrés

URI : https://id.erudit.org/iderudit/017770ar

DOI : https://doi.org/10.7202/017770ar

Aller au sommaire du numéro

Éditeur(s)

Faculté de théologie et de sciences des religions, Université de Montréal

ISSN

1188-7109 (imprimé)

1492-1413 (numérique)

Découvrir la revue

Citer ce document

Nouss, A. (2007). La traduction des textes sacrés. Théologiques, 15(2), 5-13.

https://doi.org/10.7202/017770ar

Tous droits réservés $\odot$ Faculté de théologie et de sciences des religions, Université de Montréal, 2007
Ce document est protégé par la loi sur le droit d'auteur. L'utilisation des services d'Érudit (y compris la reproduction) est assujettie à sa politique d'utilisation que vous pouvez consulter en ligne.

https://apropos.erudit.org/fr/usagers/politique-dutilisation/
Cet article est diffusé et préservé par Érudit.

Érudit est un consortium interuniversitaire sans but lucratif composé de l’Université de Montréal, l'Université Laval et l'Université du Québec à Montréal. Il a pour mission la promotion et la valorisation de la recherche. https://www.erudit.org/fr/ 


\section{La traduction des textes sacrés}

Alexis Nouss

Département de linguistique et de traduction Université de Montréal

Il se tient devant, hésitant. Il sait qu'il doit entrer mais il ignore jusqu'où il peut pénétrer. Il a certes reçu des instructions mais celles-ci seront-elles suffisantes pour éclairer ses choix en cas de besoin? De surcroît, il doit rendre compte de l'expérience à d'autres : il a précisément pour mission de rapporter les résultats de son avancée dans l'espace intérieur, plutôt que de les garder pour lui. Espace du dedans, du sacré, certes, mais combien sacré? Que sait-il, d'ailleurs, du sacré, dans la mesure où celui-ci ne saurait se divulguer? Et puis il se demande aussi comment l'espace pourrait demeurer sacré alors que lui l'aurait foulé. Du dehors, il distingue des sons, perçoit des couleurs. Celles-ci lui semblent familières, bien qu'elles ne devraient pas, puisque ce monde-là est en principe radicalement différent de ce monde-ci, le sien.

Il eût fallu la plume de Kafka dont ces lignes maladroites s'inspirent pour présenter efficacement les affres d'un traducteur confronté à un texte dit sacré. Pro-fanum (devant le temple) : face à sa tâche comme face au texte, le traducteur est irrémédiablement profane et suspect de possible profanation. Il entretient avec l'original un rapport d'extériorité, un peu comme un croyant se sentirait face au divin. Quand bien même serait-il totalement athée, il ne pourrait négliger la charge de sacralité que revêt le texte auquel il s'attache dans l'économie de sa réception, ce qui le distingue des autres formes scripturaires, y compris de celles du domaine littéraire. Si la traduction biblique a souvent servi en Occident de modèle à la théorisation de l'acte traductif, il n'empêche qu'elle demeure soumise à des paramètres qui lui sont exclusifs, problématique qui constitue la préoccupation du présent numéro.

Dans un ouvrage qui n'a rien perdu de sa pertinence, L'homme et le sacré, Roger Caillois (1972) distinguait deux formes de sacré: le sacré de respect, qui commande les interdits et le sacré de transgression, qui autorise

(C) Revue Théologiques 2007. Tout droit réservé. 
la fête. Un troisième type se présente dans le cadre de notre propos: le sacré de traduction qui tire ses éléments caractéristiques des deux précédents, le premier cherchant à préserver un ordre jugé efficace et légitime, le second prenant acte que tout ordre a besoin d'être régénéré pour se perpétuer. Le sacré de traduction veut maintenir la puissance signifiante d'un texte, nourrie de sa transcendance, tout en le soumettant à un formalisme satisfaisant les besoins immanents d'énonciation: donner, en somme, une histoire à l'éternité.

On pourra s'étonner, au demeurant, que la traduction des textes sacrés n'ait pas été traitée par le grand sociologue que fut Caillois. La réponse tient peut-être au fait qu'il prenait heuristiquement en compte une séparation nette du sacré et du profane, renforcée et non effacée par la transgression, et dont la traduction, fidèle à sa nature subversive, vient brouiller la polarité. Elle n'opère pas un saut du sacré au profane, mais un glissement que le théologique autorise et qu'une approche non confessionnelle doit aussi reconnaître et analyser.

Le sacré prend toujours des formes qui ne lui appartiennent pas afin de se dévoiler, de même que l'absolu divin doit admettre un processus de transformation ou d'émanation permettant le passage du transcendant à l'immanent. La sacralité d'un texte est-elle une catégorie particulière? Si le traducteur traduit d'abord le sacré du texte avant de traduire le texte sacré, c'est que le sacré est une dimension inhérente au texte, soit qu'on la juge provenant du texte lui-même — Luther bâtit la Réforme sur cette lecture —, soit qu'on lui attribue une origine externe - ce que font les approches historiques et sociologiques. Henri Meschonnic, pour sa part, dans un texte justement intitulé "Traduire le sacré ou traduire le rapport au divin », voit dans la Bible une poétique spécifique, fondée sur une "oralité codifiée " $(1999,428)$ qui marque un certain rapport au divin.

Le sacré, toutefois, pour être sacré, n'en est pas moins sensible à la contingence et aux variations climatiques: il est un sacré du désert comme il en est un des montagnes, une sacralité née de la mer et une autre de la fertilité terrestre. Le texte sacré connaît une similaire diversité qui correspond à ses usages et il demande une typologie. À défaut de la dresser ici, donnons deux exemples de fonctions remplies par le texte sacré: un rituel mortuaire et une visée pédagogique. Le Livre des morts égyptiens était placé dans le cercueil pour aider à la résurrection dans l'au-delà, tandis que la récitation du Livre des morts tibétain prépare à une meilleure réincarnation ou à la sortie du cycle. Quant aux treize ouvrages classiques de Confucius, sacralisés 
par décision impériale, ils furent gravés sur 190 stèles (avec plus de 600000 caractères), afin d'en perpétuer les enseignements auprès des générations successives d'étudiants de l'institution collégiale de l'Empire chinois.

Le texte sacré affiche en fait une double sacralité, dans la mesure où au sacré attribué à sa provenance ou opéré par sa réception - en ce sens comparable à celui dont peuvent être investis des objets ou des gestes dans les pratiques rituelles - s'ajoute celui de sa nature langagière qui, pour ce qui est des traditions spirituelles traitées dans le présent numéro, renvoie à une faculté humaine qui fait le lien avec la transcendance divine ou avec une réalité autre que celle du quotidien. Que ce soit le Verbe du Créateur ou la nature magique de la parole, un énoncé langagier, oral ou écrit, participe d'un code jouissant d'un statut particulier parmi les dispositifs sémiotiques.

Si l'on admet le postulat herméneutique selon lequel l'acte traductif ne s'attache pas simplement au texte (sens et forme), mais qu'il doit prendre en compte la situation socioculturelle et la tradition interprétative dans lesquelles il est entrepris, la traduction du texte sacré inclut une troisième dimension qui entraîne sa spécificité parmi les pratiques traductives, à savoir l'horizon et le dispositif de réception qui le rendent sacré. Une telle procédure de sacralisation textuelle varie selon les courants théologiques, les institutions religieuses, les langues et les codes en présence. Cette diversité est rarement considérée dans les pensées herméneutiques, y compris celles qui sont liées à des traditions religieuses qui prennent généralement la sacralité textuelle pour acquise ${ }^{1}$. Elle devrait, par ailleurs, intéresser les approches non confessionnelles, puisque, en dernier regard, le sacré est une forme de mise en discours. Cette dernière expression n'est pas innocente puisque, lorsque dans la préface de Naissance de la clinique, Foucault dénonce la méthodologie du commentaire face aux productions discursives, supposant $u n$ message à saisir sans que toutefois le signifié ne s'y épuise, il la fait remonter à sa source historique qui est, selon lui, l'exégèse du texte révélé. Si l'analyse des discours doit se garder de tomber dans l'impasse du commentaire, il importe que le modèle de celui-ci n'inspire pas une démarche traductive faisant l'économie des cadrages historiques produisant le texte sacré en tant que tel.

Ceci nous permet de préciser un point sur l'usage de la notion d'herméneutique de la traduction, qui est généralement citée pour ne nommer que la part d'interprétation dans le processus traductif. La traduction, loin

1. Paradoxalement, les écoles mystiques sont souvent plus attentives à cette question et examinent la nature des dispositifs de sacralisation afin d'en contrôler les modalités. 
de venir paresseusement illustrer la démarche herméneutique, doit se présenter en elle-même comme une situation ou un scénario interprétatifs parmi les activités de compréhension (le commentaire littéraire, l'enquête anthropologique, l'exégèse religieuse, la cure analytique, etc.) permettant d'appréhender ce qui est en jeu dans le geste herméneutique. Sa spécificité, qui est de fonctionner au sein de la multiplicité des langues, en fait un champ d'étude des plus riches pour examiner la visée de l'herméneutique qui n'est pas de dégager du sens mais, selon l'expression de Gerald R. Bruns, de pratiquer « une phénoménologie du milieu [phenomenology of the between ${ }^{2}$ ] $(1992,11)$, en s'attachant à déterminer ce que signifie «comprendre " par la comparaison de différentes situations de compréhension. Le texte sacré offert à l'interprétation ou à la traduction affiche une dimension caractéristique, sa sacralité - ou plutôt sa sacralisation - , qui influence le processus de compréhension qu'il suscite et qui fait tout l'intérêt de ce dernier pour enrichir la définition du geste herméneutique.

À parcourir les articles du numéro, il n'échappera pas au lecteur qu'ils sont tous marqués d'un certain ton que l'on pourrait qualifier d'engagé si l'on ne craignait que ce terme ne soit associé à un manque de rigueur et à une partialité dérogeant à la règle universitaire. Il ne s'agit pas d'un quelconque militantisme, mais de la marque d'un investissement personnel de la part du chercheur. La première personne semble affleurer sous les analyses — sans que leur contenu n'en soit invalidé, au contraire, et sans qu'une appartenance religieuse ne soit forcément affirmée. Il demeure qu'une part de subjectivité colore les réflexions ou les expériences dont les textes exposent le matériau et ce, selon différentes modalités : opinions affirmées avec vigueur (Gignac, Nouss), témoignage direct sur les circonstances et les conditions d'une pratique (Martinez, Legros), volonté d'infirmer de fausses représentations (Kaufmann, Basalamah).

Trois explications peuvent s'appliquer à une telle posture discursive. La traductologie, d'une part, est familière de la subjectivité depuis qu'elle s'est affranchie de la scientificité présumée que lui procuraient la linguistique et la stylistique comparées pour oser interroger l'activité traductive en tant que pratique révélatrice d'enjeux identitaires, culturels et politiques. La

2. Nous avons résisté à traduire par "phénoménologie de l'entre-deux », car la binarité est ici à proscrire pour ses dangers de totalitarisation. Le regard phénoménologique appliqué à l'herméneutique prendra en compte plus de deux paramètres (du type: sujet et objet), de même que le mouvement de la traduction ne se réduit pas à une simple bilatéralité entre deux langues, deux textes ou deux cultures. 
candeur de la croyance en un sens universellement transmissible et communicable au-delà des formes langagières et des énonciations subjectives n'est plus de mise. D'autre part, la fonction première du texte sacré est d'éclairer la question de la fondation, posée au niveau tant collectif qu'individuel, et celle-ci est suffisamment anxiogène chez l'humain pour que toute tentative de réponse - et le texte sacré en est peut-être la plus représentative - empêche tout détachement à son égard, sans sacrifier la distance du travail scientifique. La centralité persistante des textes sacrés dans les cultures les plus sécularisées témoigne aisément de la force d'inspiration qui les habite et l'histoire de l'art en fournit des exemples récurrents.

Enfin, le sacré, de par l'ambiguïté qui le nourrit et qui dialectise les notions de pur et d'impur ou de sainteté et de damnation entraîne la création sur le plan lexical de ce que nous nommerons des translatogènes, à savoir des signifiants dont le noyau sémantique ne peut se décliner qu'amphibologiquement et qui obligent donc leur usage à être une traduction dans un sens ou dans l'autre, reconduisant une intraduisibilité première participant de leur valeur signifiante. "Et si religio restait intraduisible? », a demandé Derrida $(2001,48)$ en dégageant la double dimension latine et chrétienne encadrant le discours sur et du religieux tel que l'Occident le perçoit. Si l'indécidabilité ${ }^{3}$ en vient à se confondre avec le geste même de la déconstruction et soutient le travail philosophique dans tous les domaines, celui qui circonscrit les économies des religions ou des spiritualités lui offre un matériau très fertile et comment en serait-il autrement, puisque ces positionnements tablent d'emblée sur une dimension parallèle à la matérialité du monde et invérifiable dans les termes forgées pour l'intelligibilité de celle-ci ? L'anthropologie s'est ainsi souvent interrogée sur la nature ambivalente des notions de mana et de tabou. Le Vocabulaire des institutions indo-européennes de Benveniste (1969) souligne sur différentes entrées du lexique spirituel la coexistence d'interprétations divergentes ou contradictoires. Sacer, sacré et maudit à la fois, ne pouvant donc être touché, est un exemple connu ${ }^{4}$. Le vocabulaire grec de la souillure et de l'expiation en recèle d'autres 5 .

3. Les indécidables sont dans la pensée derridienne ces termes ou notions dont le sens résiste à la fixation binaire (ceci ou cela) de même qu'ils résistent au dépassement vers un palier résolutoire.

4. Pour une relecture récente de la notion de sacré et sa réinterprétation dans une perspective politique, voir Agamben (1997).

5. Voir Caillois (1972, 40-41). Caillois cite également les domaines linguistiques polynésien, amérindien et japonais quant à la désignation du surnaturel. 
Ici encore, le regard du traducteur se révèle unique dans sa capacité à défier les ethnocentrismes de tous genres: les catégories construites autour du fait religieux en Occident ne peuvent être déplacées ailleurs sans perte ni dommage. Ceci a des conséquences sur le plan sociopolitique en Occident même, puisque désormais l' "autre religieux" — qui dévoile aussi un autre du religieux, si cette notion appartient en propre à l'Occident - ne se trouve plus uniquement ailleurs mais au sein des sociétés européennes et américaines.

On ne s'étonnera plus que les articles du présent numéro ne se contentent pas d'un examen normalisant les problématiques liées à la traduction des textes sacrés, mais qu'ils les interrogent dans leur fondement même et dans les représentations qui les accompagnent, sans craindre le « déboulonnage » de certaines certitudes, voire l'émission de propositions audacieuses.

Francine Kaufman s'attache à étudier l'attitude du judaïsme de l'Antiquité à l'endroit de la traduction du texte de la Torah afin de dénoncer la fausse conception selon laquelle le judaïsme en prononcerait la condamnation, un cliché qui accompagne la traductologie, même parmi ses théoriciens les plus éclairés. Interrogeant la littérature exégétique à travers son regard traductologique et la donnant à lire par de nombreuses citations, elle montre comment le rituel synagogal intégra la traduction, qui trouvait ainsi sa place depuis l'épisode de la Révélation jusqu'à la lecture publique de la Torah. La langue vernaculaire ne souffre d'aucun discrédit, tandis que traduction et interprétation reçoivent une conceptualisation bien précise, s'appliquant à l'ensemble des Livres formant le canon juif du Premier Testament (la Torah, les Prophètes, les Hagiographes). Ce sont aussi les statuts respectifs de l'écriture et de l'oralité et les procédures de légitimation qui sont examinés à partir de divers exemples, notamment les deux «traductions écrites autorisées », celles d'Onkelos et d'Aquila, qui illustrent l'accueil favorable des langues autres que l'hébreu. L'histoire de la réception de la Septante par le judaïsme rabbinique est enfin reprise afin de réévaluer la thèse de son rejet absolu. En dernier regard, le souci du judaïsme porte sur l'ouverture du texte révélé à tous ceux qui sont susceptibles de le recevoir et la traduction, dans une logique de "co-présence », en participe assurément.

L'article d'Alain Gignac se veut une réponse à mon article 6 , en un geste rhétorique qui sied à toute interrogation sur le religieux, puisque Benveniste

6. Qu'on me permette de ne pas gloser sur ma propre réflexion dans le cadre de ce liminaire et de renvoyer le lecteur au résumé de mon article. 
rappela que la racine spend (d'où respondere) revêt une grande importance dans le vocabulaire religieux. S'appuyant sur l'expérience de sa participation à l'entreprise de La Bible, nouvelle traduction (BNT) et empruntant le point de vue de la théologie chrétienne, Alain Gignac réfléchit sur la possibilité de validation d'une "bonne traduction chrétienne». Il revient pour cela sur l'ambition de la BNT de redonner à la Bible sa capacité d'être lue, en accord avec la sensibilité contemporaine attachée à la production littéraire. Ce que visait la collaboration entre un exégète et un écrivain relevait de la volonté d'entendre à nouveau le texte biblique dans son actualité, sa capacité à parler une langue d'aujourd'hui, la rencontre des deux collaborateurs anticipant, d'une certaine manière, la rencontre souhaitée entre le lecteur et le texte et assurant l'équilibre entre texte-source et texte-cible. Après la description des difficultés d'un geste herméneutique qui considère le texte comme un organisme vivant et sa lecture comme un dialogue - d'abord avec soimême - les images prenantes d'une randonnée en forêt ou de la visite d'une cathédrale aident Alain Gignac à préciser la redéfinition du sacré qu'il propose en le déplaçant du côté de la réception — l'humaine réception - et non plus de la source. Ceci lui permettra en conclusion de n'accepter la notion de texte sacré que si celle-ci implique une expérience de foi, quelle que soit la langue dans la prolifération admise des traductions.

Pour Gaafar Sadek et Salah Basalamah, la question de la traduction du Coran, si elle se pose sur le plan théologique, n'est pas moins riche de données à analyser dans une triple perspective, historique, sociologique et traductologique. Traduire le texte coranique se pose comme nécessité en raison de la dynamique expansive de l'islam dès ses débuts, le Livre révélé en arabe circulant auprès de populations non-arabophones. Juristes et théologiens se sont affrontés sur la question, d'autant que tout au long de l'histoire, des traductions ont été produites à des fins polémiques. Passant en revue de nombreuses traductions en diverses langues et à diverses époques, les auteurs articulent avec une grande précision analytique les notions permettant d'évaluer la justesse de ces entreprises: le souci interprétatif, le poids politique, l'exactitude linguistique. Un concept particulier à la tradition coranique, celui de l'inimitabilité du texte sacré, vient jouer en regard de la visée universaliste portée par le message révélé et oriente les termes du débat: nature et statut de la langue arabe, rapport de la forme et du sens, usages du texte coranique dans la liturgie, représentations et fonctions respectives du texte révélé et de ses traductions. En toute rigueur herméneutique, un triple mouvement vient interroger le Coran lui-même, les conditions actuelles de sa réception et la théorie littéraire moderne pour 
énoncer une thèse dialectisant la sacralité coranique et le devenir historique de l'identité musulmane.

L'article de Nicole Martínez-Melis présente le double intérêt de livrer une réflexion théorique sur la traduction des textes bouddhiques et d'informer sur la pratique traductive elle-même, telle qu'elle est menée au sein de son équipe. Le cadre disciplinaire accueillant ce travail relève à la fois de la terminologie et de la traductologie et il est utilisé pour montrer combien les horizons d'attente peuvent brouiller ou occulter une réelle compréhension du bouddhisme tibétain. Une première typologie est dessinée qui adopte une perspective diachronique afin de regrouper en continuité les textes fondateurs et les enseignements actuels, rejetant la notion de texte sacré comme non applicable; une seconde étudie les motivations traductives fort divergentes selon les cadres de réception. Cette dernière diversité demande à examiner et à classifier les entreprises traductives selon des critères linguistiques, traductologiques et pragmatiques. L'anthropologie sociale et culturelle est invoquée pour déjouer les pièges de l'assimilation ou de l'exotisation et proposer une troisième voie méthodologique, la "transculturisation ", afin d'échapper au stérile dilemme entre préoccupations sourcière et cibliste. La démarche du groupe de recherche et de traduction MarpaTerm a abouti à la constitution d'une banque de données terminologiques visant à la standardisation du lexique de la tradition tibétaine dont les techniques de traduction sont ici présentées.

Dominique Legros met pareillement en garde contre l'effacement des différences particularisant les religions autochtones qui menace la traduction culturelle la mieux intentionnée. L'article commence par proposer un exercice de défamiliarisation et de redéfinition à l'endroit de différentes catégories connues du religieux: le principe de séparation entre deux mondes, le monisme opposé au dualisme, la présence des divinités, les structurations généalogique et cosmologique, les dimensions de la temporalité. Puis, s'attachant au cas des Tuchtone du territoire du Yukon, l'anthropologue expose les principes d'une religion «sans dieu » à partir des exemples de la création du monde et du rôle des figures primordiales entre règnes animal et humain, ainsi que de la considération des rapports entre vie et mort. C'est la narration ethnographique qui prend le relais avec le récit de scènes vécues (une nuit à -50 degrés, un épisode de chasse, etc.) et l'examen traductologique de signifiants centraux dans la culture des Tuchtone intégrant des pratiques chamaniques et magiques, notamment: le $z h a ̈ a k$, une forme de pouvoir et de cognition, le yindi', un principe d'intelligibilité, le yunzrun', l'ombre-âme. L'article défend en conclusion la possibilité de concevoir une 
religion sans transcendance et même sans surnaturel, mais qui pourtant organise un système de croyances tout à fait cohérent et légitime.

C'est à ma connaissance la première fois que la traductologie francophone accueille une réflexion sur le texte sacré s'ouvrant à la fois à l'Orient, au monde autochtone et à l'oralité, et regroupant par ailleurs les trois monothéismes dans une orientation méthodologique faisant une part égale à la théorisation et aux considérations pratiques. Que la direction et le comité de rédaction de la revue Théologiques soient remerciés pour cette occasion donnée et son secrétariat de rédaction félicité pour l'admirable travail de préparation et de réalisation du numéro. Un grand merci également à Jenna Smith pour son assistance à la traduction.

\section{Références}

Agamben, G. (1997), Homo sacer. Le pouvoir souverain et la vie nue / trad. par M. Raiola, Paris, Seuil.

BENVENISTE, É. (1969), Le Vocabulaire des institutions indo-européennes, Paris, Minuit.

Bruns, G.R. (1992), Hermeneutics Ancient and Modern, New Haven / London, Yale University Press.

CaILlois, R. 1972 [1950], L’homme et le sacré, Paris, Gallimard (Idées).

Derrida, J. (2001), Foi et savoir, Paris, Seuil (Points/Essais).

Meschonnic, H. (1999), Poétique du traduire, Lagrasse, Verdier. 